\title{
Thermopile Arrays for IR Imaging and body temperature screening applications
}

\author{
Schieferdecker, J.; Schnorr, M., Forg, B.; Herrmann, F., Schmidt, C.; Leneke, W.; Simon, \\ M. Heimann Sensor GmbH, Maria-Reiche-Str. 1, 01109 Dresden, Tel. +49-351-888885-0; \\ E-mail: info@heimannsensor.com
}

\begin{abstract}
Infrared arrays found their way into wide spread applications in various industries. Due to increasing resolutions and decreasing costs the growth rates for Infrared (IR) imaging sensors and cameras are assumed to continue having double digit annual growth rates also for the coming decade of the twenties. While photon IR detectors have been the drivers for thermal imaging in last century, the innovations in Si microelectronics and MEMS paved the way of success for uncooled thermal detector arrays. Pyroelectric arrays, which lead to the very first automotive night vision camera with 80000 pixels in year $2000 / 1 /$, fell back due to their need of continuous mechanical modulation and the difficulty to integrate pyroelectric sensitive materials into monolithic CMOS structures. After that, micro-bolometers dominated the high resolution imaging markets /2/; while fully monolithic Poly-Si type IR thermopiles where the trendsetters for lower resolution consumer applications.
\end{abstract}

Unlike the other array technologies the thermopile arrays allow to build true shutterless radiometric IR cameras. The reason is, that thermopile arrays are DC sensitive devices and do not need to be biased. Thermopile arrays with pixel numbers from $8 \times 8,16 \times 16,32 \times 32$ resp. $32 \times 24$ were introduced to mass production for various consumer applications. In addition, the pixel size of $90 \mu \mathrm{m}$ allowed a first thermopile array with $80 \times 64$ pixels $/ 3 /$.

Especially the $32 \times 32$ arrays and the $80 \times 64$ found large acceptance for automatic fever screening applications in the fight against the spread of actual COVID-19 worldwide pandemic.

In first part of this paper we describe, how fast the lower resolution thermopile arrays could be integrated into body temperature screeners to fight against COVID-19 pandemic spread. Second part will introduce first thermopile arrays with $60 \mu \mathrm{m}$ pixel size, which allow to extend the application range into thermal Imaging and surveillance. All necessary signal conditioning and readout electronics including SPI interface are monolithically integrated on the sensor chip and allow thermopile arrays up to $120 \times 84$ pixels to fit in a standard TO-8 housing.

Most thermopile arrays going into the body temperature screeners using simple $32 \times 32$ arrays with a single $\mathrm{Ge}$ lens using special coating for the 8-14 $\mu \mathrm{m}$ range. Due to small chip, simple fixed focus optics and no need for vacuum packaging, they can be produced in very high volumes. Due to low pixel count, the screening was only for one person in narrow range up to about $1 \mathrm{~m}$ or so - sufficient for building entrance control. 80x64 arrays were bigger and more costly, but could be used to measure temperatures at up to three or four persons simultaneously.

The digital output via SPI interface reduces the number of necessary connections of both $80 \times 64$ and the new $120 \times 84$ arrays to 6 -pin only. Thanks to integrated 16 Bit AD converters onchip the sensor arrays can be operated with Frame Rates up to $12 \mathrm{~Hz}$ (full resolution) and allow a very wide dynamic range with object temperatures up to $1000{ }^{\circ} \mathrm{C}$. Higher frame rates are possible by setting the ADC resolution to 15 or 14 Bit. Since the new $120 \times 84$ array chip has $60 \mu \mathrm{m}$ pixels vs the $90 \mu \mathrm{m}$ pixels of the $80 \times 64$, both chips come with similar focal plane and chip sizes. Due to their identical SPI interface both chips can be mounted in same housing with same optics, allowing a fully compatible "drop in" solution.

Thermopile arrays are low cost but efficient sensors in 2020 fighting the COVID-19 

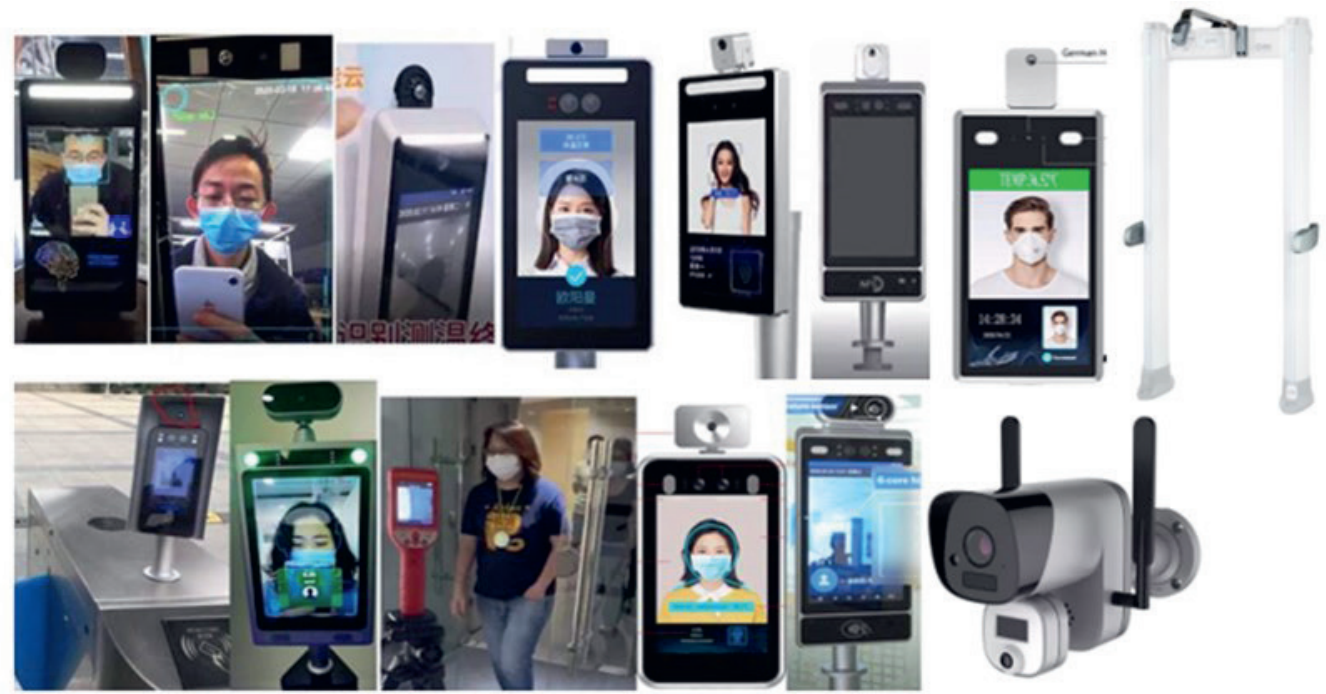

Fig. 1 shows a selection of devices for body temperature measurement in the market.
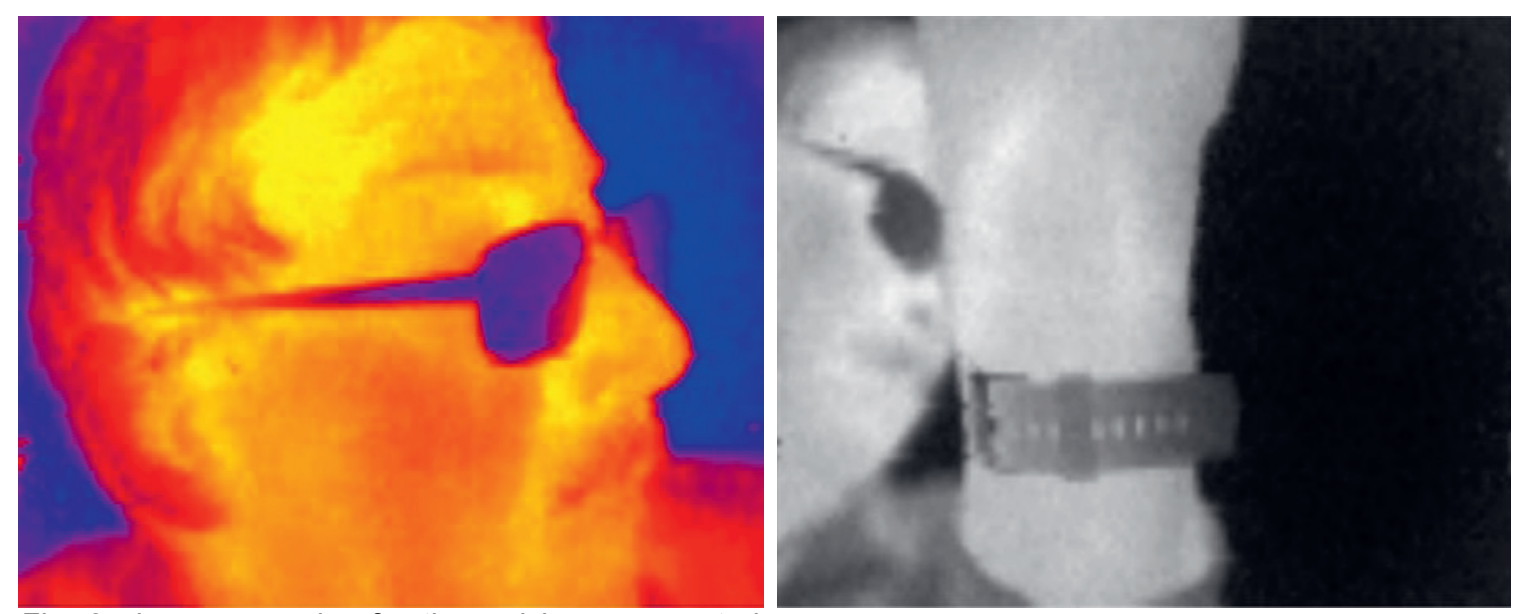

Fig. 2 shows examples for thermal images created by the $120 \times 84$ array module.

\section{References}

[1] Martinelli, N., Boulanger S.: "Cadillac de Ville Thermal Imaging Night vision system", SAE Technical Paper 2000-01-0323, 2000

[2] T. Hoelter, A. Kathman, A. Richards, M. Walters; "Key Technology Trends and Emerging Applications for Compact Thermal Imagers", Proceeedings, Conference Sensor \& Test, Nuremberg, 2015, p. 938.

[3] Schieferdecker, J.; Schnorr, M., Forg. B.; Herrmann, F., Schmidt, C.; Leneke, W.; Simon, M.: A new family of digital Thermopile Arrays for high volume applications; Proceedings Conference Sensor \& Test 2019, Nuremberg, Germany 